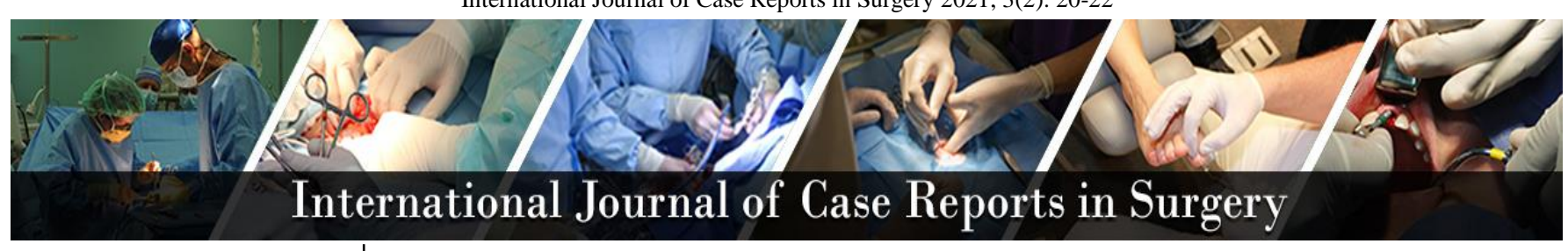

E-ISSN: 2708-1508 P-ISSN: 2708-1494 IJCRS 2021; 3(2): 20-22 www.casereportsofsurgery.com Received: 12-06-2021 Accepted: 14-07-2021

Luai Farhan Zghair College Of Medicine, Al- Iraqia University, Baghdad, Iraq

Jamal Khalil AL-Qaisy College Of Medicine, Al- Iraqia University, Baghdad, Iraq

Mohammed Hussein Mushrif College Of Medicine, Al- Iraqia University, Baghdad, Iraq
Corresponding Author: Luai Farhan Zghair College Of Medicine, Al- Iraqia University, Baghdad, Iraq

\section{Intestinal obstruction due to non-hodgkin lymphoma, interventional case study}

\author{
Luai Farhan Zghair, Jamal Khalil AL-Qaisy and Mohammed Hussein \\ Mushrif
}

DOI: $10.22271 / 27081494.2021 . v 3.12 a .29$

\begin{abstract}
Lymphoma is almost always treated, and patients usually live a long time after being diagnosed. Lymphoma comes in a variety of forms. It can induce a range of symptoms regardless of the type and location in the body. Nevertheless, some patients have no symptoms and their lymphoma is discovered through examinations for another illness. Lymphoma symptoms can be caused by a variety of factors. Having one or more of these symptoms does not always mean you have lymphoma. Several patients, on the other hand, have no signs and their lymphoma is discovered during examinations for a different illness. Other conditions can cause lymphoma symptoms. You don't necessarily have lymphoma if you have one or more of these symptoms. Lymphoma might cause you to catch infections more readily and have trouble getting rid from them. With lymphoma, sweats can happen at any point of day, but they are more prevalent at night. They're commonly described as 'drenching,' and they can soak your nightclothes or bed sheets. Lymphoma can cause itching ('pruritus') without causing a rash. It can be really inconvenient, especially when it gets hot. Fever (temperatures above $38^{\circ} \mathrm{C}$ or $100.4^{\circ} \mathrm{F}$ ) affects some persons. Fevers are frequently associated with night sweats and weight loss, but they can also occur alone.
\end{abstract}

Keywords: Lymphoma, symptoms, nodes

\section{Introduction}

The lymphatic system consists of lymph nodes and veins that carry lymphatic fluid throughout the body. Lymphatic vessels are a vast and intricate network from channels that originate as "porous" blind-ended lymphatic capillaries in the body's tissues and converge to create a number from high vessels that finally unite with enormous veins in the root of the neck ${ }^{[1]}$. Lymphatic arteries primarily throughout nutrient transport, absorb fluid lost from vascular capillary beds activities and return it to the vascular system's venous branch. 1Pathogens, lymphocytic cells, cell products, and cell debris are all present interstitial fluid, which flows into lymphatic capillaries. 1. Certain fats absorbed and processed packed to protein-coated fat droplets by the intestinal epithelium (chylomicrons) in the small intestine, which are removed from endothelial cells and enter the interstitial compartment, where they are supplied to the venous system in the neck, including other interstitial fluid elements. 1 The lymph vessels is thus a main route from transport for lipid ingested by the gut; the current in greatest lymphatic vessels is pure and is recognized as lymph; the fluid loaded by lymphatic vessels of the small bowel is opaque and milky due to the prevalence from chylomicrons and is identified as chyle; lymphatic vessels are found in almost every part of body except the brain, bone marrow, and avascular tissues such as epithelia and cartilaginous; and there are lymphatic Glands are encapsulated formations that block the flow from lymphatic veins and include parts from the body's defense system, such as clusters of lymphocytes and macrophages. They operate as complex filters, trapping and phagocytizing lymphatic particulates that passes past them. Although Lymphocytes are excellent filters with a low flow rate, cells that metastasis from essential tumors and enter lymphatic arteries frequently lodge and proliferate as secondary cancers in lymph nodes. Lymphocytes that drain sick or diseased areas can expand or alter physically, like becoming hard or painful. These changes can be utilized by physicians to detect pathologic changes or track disease spread. A variety from body areas are linked to clusters or an abundance of lymph glands ${ }^{[1]}$. Lymph nodes are plentiful and palpable in the axilla, groin and femoral region, and neck; deep locations that are not palpable include those linked with the trachea and bronchi in the thorax, and the aorta and its branching in the abdomen.1Lymph nodes in some systemic illnesses, the liver might become diffusely enlarged., or local organizations may get enlarged with main lymph node cancers, like lymphoma ${ }^{[1]}$. 
Patient And Method: In our study 6 years old childe presented with nausea, vomiting, abdominal distention, and constipation and on examination, the patient was pale, pulse rate 110 beat per minute, blood pressure $80 / 50 \mathrm{~mm} \mathrm{HG}$, temperature $39{ }^{\circ} \mathrm{C}$, respiratory rate 20 per minute, chest examination no abnormality diagnosed, The abdominal examination revealed abdominal distention, tenderness, resonance on percussion, and the bowel sound was negative. The investigation were done revealed

HB: $10.1 \mathrm{GM} / \mathrm{dl}$

PCV: $30.8 \%$

PLT: 491,000/C.MM

WBC: 15,300 CELLS/C.MM

\section{Blood Film}

Normochromic, Normocytic, Macrocyte, Anisocytosis, Leukocytosis, Thrombocytosis.

\section{Ultrasound of Abdomen}

Hepatomegally, Non Homogenous Texture, Loaded With Multiple Irregular Hypoecheoic Masses, With Diffuse Sever Bowel Wall Thickining Involving Multiple Bowel Loops Reaching The Rectum Associated With Large Upper Abdominal Solid Mass.

\section{Ct scan of Abdomen}

Extensive Rounded Masses All Over The Abdomen With Ascites And Bowel Distention.(Intestinal Obstruction With Multiple Abdomina Masses).

Operation: Midline laparotomy was done and about $600 \mathrm{ml}$ of peritoneal fluid was evacuated from the peritoneal cavity, and multiple and generalized abdominal lymphadenopathy were founded with intestinal obstruction, right sided hemicoloctomy with end to side ileo- transverse anastamosis with transverse colon vented colostomy were done to patient, were fixed in the right side of the upper abdomen, The tissue send for hitopathology and non-Hodgkin's lymphoma (NHL) was diagnosed, so patient send for specialist in radio chemotherapy and after completed the radio chemotherapy courses after one year closure of the vent- colostomy was done to the patient.

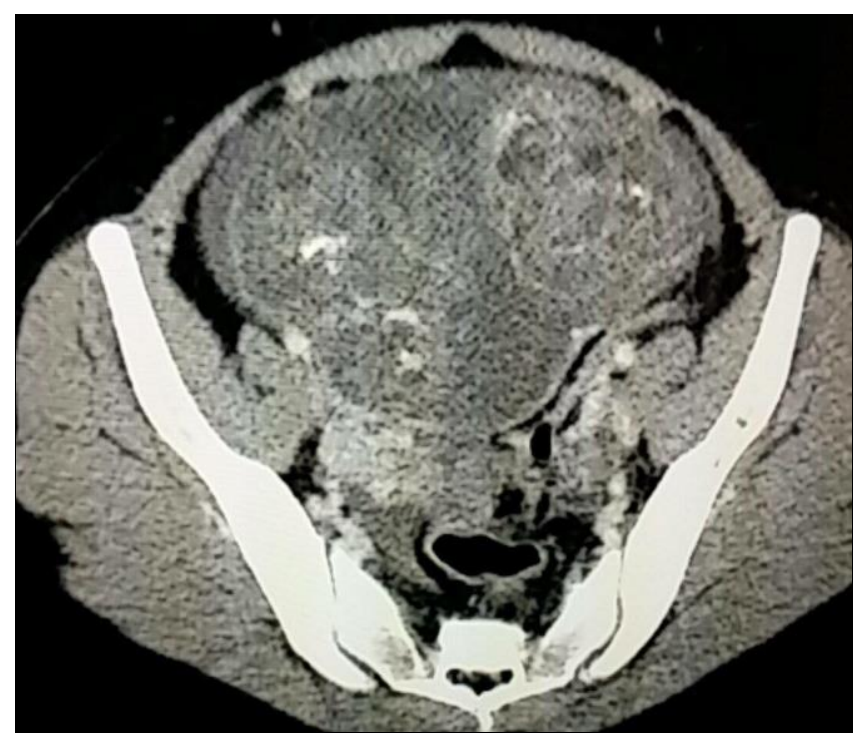

Fig 1: CT scan of the abdomen showed multiple masses (Transverse Section)

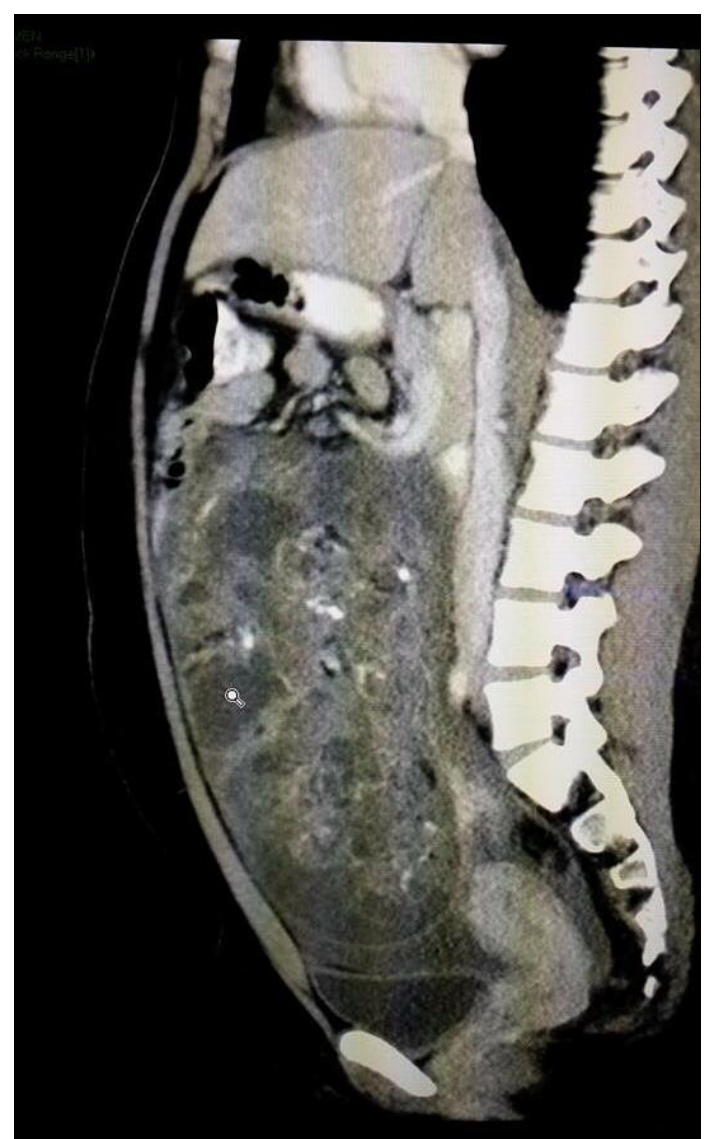

Fig 2: CT scan of Abdomen showed multiple masses (Sagittal Section)

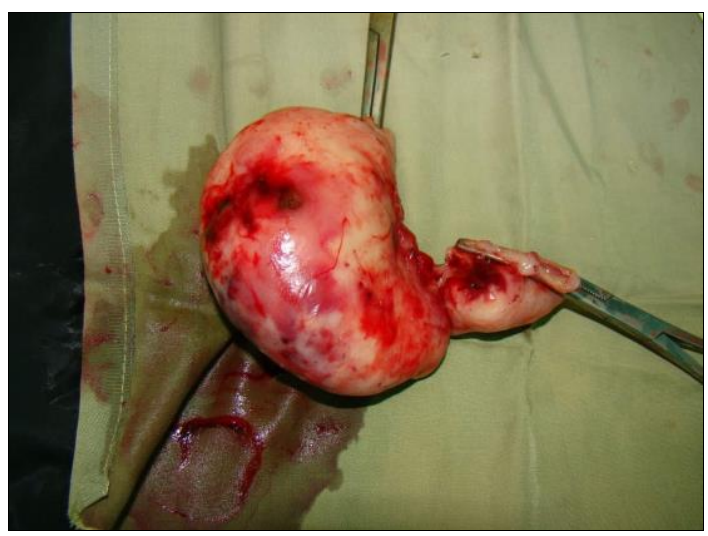

Fig 3: Mass removed from the abdomen and send for hitopathology

\section{Discussion}

The lymph vessels normally defends the body, but lymphocytes, or white blood cells, can become cancerous. Lymphomas are the designations for malignant tumors that develop in the lymphatic system. Lymphoma is a form of blood cancer that can strike at any age. It is the fifth most frequent cancer in the United Kingdom. 2Lymphomas any portion of the lymphatic system, including the spleen and lymph nodes, might be affected. (Bone marrow, thymus, spleen, tonsils, lymph nodes). Non-lymphoma Hodgkin's (NHL) and Hodgkin's lymphoma (HL) are two types of lymphoma. The two most frequent kinds of lymphoma are Hodgkin's (NHL) and non-(NHL). Hodgkin's Uncontrollable cell development is the cause of cancer. Lymphomas can occur in any part of the lymphatic system, including the spleen and lymph nodes (bone marrow, 
thymus, spleen, tonsils, and lymph nodes). Hodgkin's lymphoma (HL) and non-lymphoma Hodgkin's (NHL) are the two most common kinds of lymphoma. Uncontrollable cell development is the cause of cancer. Almost $1 \%$ of all gastrointestinal neoplasms are primary gastrointestinal lymphoma. The stomach is more commonly involved than the small or large intestine, and it has a better prognosis. Only abdominal pain and weight loss may be observed, and they may last for months or years before a diagnosis is determined. Perforation and blockage are uncommon. Multiple tumors account for $8 \%$ of all patients. At laparotomy, only one-third of lymphomas are contained to the bowel. Reticulum cell sarcomas account for one-third of the cases, with the remainder being lymphosarcoma or lymphocytic lymphoma. The average five-year survival rate was $38 \%$. Curative resections resulted in a $60 \%$ chance of survival regardless of region, whereas palliative resections produced only a $17 \%$ chance of cure. Survival was indirectly linked to the amount of nodal dissemination, as expected.

\section{References}

1. GR AY'S Anatomy for Students, chapter one (the body, lymphatic system), Third Edition (International Edition), Churchill Livingstone Elsevier, 29-31.

2. Contreary K, Nance FC, Becker WF. Annals of surgery, Primary lymphoma of the gastrointestinal tract. 1980;191(5):593-598. 